\title{
PHH3 as an Ancillary Mitotic Marker in Gastrointestinal Stromal Tumors
}

\author{
Yooju Shin · Jiyeon Hyeon \\ Boram Lee · Sang Yun Ha \\ Min Eui Hong · In Gu Do \\ Kyoung-Mee Kim
}

Department of Pathology and Translational Genomics, Samsung Medical Center, Sungkyunkwan University College of Medicine, Seoul, Korea

\section{Received: August 6, 2014 \\ Revised: September 24, 2014 \\ Accepted: October 8, 2014}

\section{Corresponding Author}

Kyoung-Mee Kim, M.D.

Department of Pathology and Translational Genomics,

Samsung Medical Center, Sunkyungkwan University

School of Medicine, 81 Irwon-ro, Gangnam-gu,

Seoul 135-710, Korea

Tel: +82-2-3410-2807

Fax: +82-2-3410-6396

E-mail: kkmkys@skku.edu
Background: Counting mitoses is subjective and time-consuming. The adjunctive diagnostic utility of a recently reported mitotic marker, phosphohistone $\mathrm{H} 3(\mathrm{PHH} 3)$, was investigated in gastrointestinal stromal tumors (GISTs). Methods: We reviewed 77 GISTs for several proliferative indices. These included the mitotic count per 50 high power fields (HPFs), the immunohistochemical Ki67 labeling index and the immunohistochemical PHH3 mitotic index (MI). For comparison, Spearman's rank correlation and interclass correlation coefficient were used. Results: Mitotic counts ranged from 0-138 (mean, $7.57 \pm 2.34$ ) and the $\mathrm{PHH} 3 \mathrm{Ml}$ ranged from 0-126 per $50 \mathrm{HPFs}$ (mean, $9.61 \pm 2.27)$. We found a positive correlation between mitotic counts and $\mathrm{PHH} 3 \mathrm{Ml}(r=0.810, p<.001)$. The inter-observer correlation coefficient for three participants was 0.975 for mitotic counts and 0.940 for the $\mathrm{PHH} 3 \mathrm{Ml}$. When using the $\mathrm{PHH} 3 \mathrm{Ml}$ instead of mitotic counts in the Armed Forces Institute of Pathology (AFIP) stratification criteria, 10 cases were reclassified. In one patient with a mitotic count of 2 and a PHH3 Ml of 6 per 50 HPFs, distant metastasis occurred. Conclusions: In GISTs, the PHH3 MI correlated adequately with mitotic counts and can be used as a useful adjunctive to count mitotic figures efficiently.

Key Words: Gastrointestinal stromal tumors; Mitosis; Ki-67; Biological marker
Gastrointestinal stromal tumors (GISTs) are the most common mesenchymal tumors of the gastrointestinal (GI) tract. GISTs can occur anywhere along the GI tract from the esophagus to the rectum, but most are located in the stomach (60\%), jejunum and ileum (30\%), and duodenum (5\%). ${ }^{1}$ The tumors have a wide spectrum of biological and clinical behaviors. Some GISTs rarely metastasize or recur, whereas others progress rapidly. ${ }^{2}$ Great effort has been invested in establishing a histopathological classification that can predict aggressive behavior in GISTs. Franquemont ${ }^{3}$ first described differentiation and risk assessment of GISTs in 1995. In 2002, the National Institutes of Health (NIH) consensus criteria used tumor size and mitotic counts as key prognostic factors. ${ }^{4}$ Recently, data from the Armed Forces Institute of Pathology (AFIP) revealed that small intestinal GISTs have higher malignant potential than gastric GISTs ${ }^{5,6}$ and the AFIP risk stratification is used widely to predict prognosis of patients with GIST. ${ }^{1}$

The most important prognostic indicator in GISTs is the mitotic count. However, counting mitoses is time-consuming and subjective which leads to high inter-observer variability. ${ }^{7,8}$ More- over, mitotic figures are indistinguishable from apoptotic figures. Phosphohistone 3 ( $\mathrm{PHH} 3$ ) is a mitotic specific marker recently evaluated in a wide array of different tumor types ${ }^{9}$ and shown to be a useful tool for counting mitoses and predicting prognosis. ${ }^{9-18}$ Several studies showed $\mathrm{PHH} 3$ enhanced rapid recognition of mitotic figures in meningiomas, which are classified based on the mitotic index (MI). ${ }^{9-11} \mathrm{PHH} 3$ was also very helpful in mitotic counting in breast carcinomas, ${ }^{12}$ melanocytic lesions, ${ }^{13}$ uterine smooth muscle tumors ${ }^{14}$ and pulmonary neuroendocrine carcinomas. ${ }^{15}$ Furthermore, $\mathrm{PHH} 3$ expression was reported to be an independent prognostic factor in gastric adenocarcinoma ${ }^{16}$ and esophageal squamous cell carcinoma. ${ }^{17}$ Recently, Kim et al. ${ }^{19}$ reported $\mathrm{PHH} 3$ staining primarily supported grading by facilitating mitotic counting and might have prognostic value in GISTs. However, inter-observer agreement in quantifying mitoses and the $\mathrm{PHH} 3 \mathrm{MI}$ has not been established. Moreover, the difference in the risk of tumor progression observed between mitotic counts and the $\mathrm{PHH} 3 \mathrm{MI}$ has not been thoroughly explored. The aim of this study was to determine the difference, if any, in the risk of tumor progression when 
using the PHH3 MI instead of mitotic counts in the established AFIP risk stratification criteria. Additionally, inter-observer agreement between mitotic counts and the $\mathrm{PHH} 3 \mathrm{MI}$ was assessed.

\section{MATERIALS AND METHODS}

Seventy-seven patients with KIT (CD117)-positive primary GISTs who underwent surgical resection at Samsung Medical Center between 2012 and 2013 were selected for this study. Patients who received neoadjuvant Gleevec therapy were excluded. The medical records of patients were reviewed for tumor size and tumor location. Mitotic figures were counted at the highest proliferative region and the mitotic counts were recorded as the number of mitoses per 50 high power fields (HPFs).

The formalin-fixed, paraffin-embedded tumor tissue from resected specimens was cut into $3-\mu \mathrm{m}$ sections and mounted on slides. The tissue section slides were then deparaffinized and immunostained using the Leica Bond-Max automatic stainer (Leica Microsystems, Bannockburn, IL, USA). All hematoxylin and eosin $(\mathrm{H} \& \mathrm{E})$-stained slides were reviewed and a representative slide was chosen containing the most mitotically-active

Table 1. Clinicopathological characteristics of the 77 patients with GIST

\begin{tabular}{|c|c|}
\hline Variable & $\mathrm{n}(\%)$ \\
\hline Median age (range, yr) & $57(28-86)$ \\
\hline $\begin{array}{l}\text { Gender } \\
\text { Female } \\
\text { Male }\end{array}$ & $\begin{array}{l}28(36.4) \\
49(63.6)\end{array}$ \\
\hline $\begin{array}{l}\text { Tumor size }(\mathrm{cm}) \\
\quad \leq 2 \\
>2 \text { and } \leq 5 \\
>5 \text { and } \leq 10 \\
>10\end{array}$ & $\begin{aligned} 8 & (10.4) \\
52 & (67.5) \\
12 & (15.6) \\
5 & (6.5)\end{aligned}$ \\
\hline $\begin{array}{l}\text { Tumor location } \\
\text { Stomach } \\
\text { Duodenum } \\
\text { Jejunum/ileum } \\
\text { Rectum }\end{array}$ & $\begin{array}{c}54(70.1) \\
12(15.6) \\
10(13.0) \\
1(1.3)\end{array}$ \\
\hline $\begin{array}{l}\text { Mitosis } \\
\leq 5 / 50 \mathrm{HPFs} \\
6-10 / 50 \mathrm{HPFs} \\
>10 / 50 \mathrm{HPFs}\end{array}$ & $\begin{aligned} 62 & (80.5) \\
4 & (5.2) \\
11 & (14.3)\end{aligned}$ \\
\hline $\begin{array}{l}\text { Ki-67 LI (\%) } \\
\quad \leq 5 \\
6-10 \\
>10\end{array}$ & $\begin{array}{r}59(76.6) \\
10(13.0) \\
8(10.4)\end{array}$ \\
\hline $\begin{array}{l}\text { PHH3 Ml } \\
\leq 5 / 50 H P F s \\
\text { 6-10/50HPFs } \\
>10 / 50 H P F s\end{array}$ & $\begin{array}{l}51(66.2) \\
11(14.3) \\
15(19.5)\end{array}$ \\
\hline
\end{tabular}

GIST, gastrointestinal stromal tumor; HPFs, high power fields; Ki-67 LI, Ki67 labeling index; $\mathrm{PHH} 3 \mathrm{Ml}$, phosphohistone $\mathrm{H} 3$ mitotic index. area. Immunohistochemical staining was then performed using monoclonal antibodies against Ki-67 (1:300, Dako, Glostrup, Denmark) and polyclonal antibodies against $\mathrm{PHH} 3$ (1:4,000, Ser10, Millipore, Billerica, MA, USA). The Ki-67 labeling index (LI) was assessed manually as the percentage of positively stained cells out of at least 1,000 tumor cells within the highest proliferative area. The number of $\mathrm{PHH} 3$-positive nuclei was calculated per 50 HPFs to attain the PHH3 MI. Three different pathologists independently reviewed the H\&E-stained slides to assess the mitotic count, the Ki-67 and the $\mathrm{PHH} 3$-immunostained slides for the Ki-67 LI and the PHH3 MI, respectively.

Statistical analysis was performed using IBM SPSS Statistics ver. 20 (IBM Co., Armonk, NY, USA). The Spearman rank correlation test was used to compare relationships between the mitotic count, the Ki-67 LI and the PHH3 MI. The inter-observer agreement for mitotic counts and $\mathrm{PHH} 3 \mathrm{MI}$ was calculated using the interclass correlation coefficient. A $\mathrm{p}<.05$ was considered to indicate statistical significance.

\section{RESULTS}

Table 1 summarizes the clinicopathological characteristics of the 77 patients. The median age of the patients was 57 years (range, 28 to 86 years) and $49(63.6 \%)$ were males. The tumor locations included the stomach $(\mathrm{n}=54)$, duodenum $(\mathrm{n}=12)$, jejunum/ileum ( $\mathrm{n}=10)$, and rectum $(\mathrm{n}=1)$. Mean tumor size was $4.39 \mathrm{~cm}$ (range, 1 to $36 \mathrm{~cm}$ ). Mitotic counts ranged from 0-138 per $50 \mathrm{HPFs}$ (mean, $7.57 \pm 2.34$; median, 2). The Ki-67 LI ranged from 1\%-25\% (mean, 4.83 \pm 0.61 ; median, 3). The $\mathrm{PHH} 3$ MI ranged from 0-126 per $50 \mathrm{HPFs}$ (mean, 9.61 \pm 2.27 ; median, 4). The risk of aggressive behavior stratified according to the NIH criteria ${ }^{4}$ and the AFIP criteria ${ }^{1}$ of the 77 tumors is described in Table 2.

A limited positive correlation was found between mitotic counts and the Ki-67 LI $(r=0.605, \mathrm{p}<.001)$ and a significant positive correlation was found between mitotic counts and the

Table 2. Risk of aggressive behavior in GISTs stratified according to the $\mathrm{NIH}$ and AFIP criteria

\begin{tabular}{lcc}
\hline Risk subgroup & NIH criteria & AFIP criteria \\
\hline Very low & $4(5.2)$ & $36(46.9)$ \\
Low & $48(62.3)$ & $22(28.6)$ \\
Intermediate/moderate & $9(11.7)$ & $12(15.6)$ \\
High & $16(20.8)$ & $7(9.1)$ \\
\hline
\end{tabular}

Values are presented as number (\%).

GISTs, gastrointestinal stromal tumors; NIH, National Institutes of Health; AFIP, Armed Forces Institute of Pathology. 
Table 3. Clinicopathological characteristics of the 10 cases stratified before and after the PHH3 Ml replaced mitotic counts in the AFIP criteria

\begin{tabular}{|c|c|c|c|c|c|c|c|c|}
\hline \multirow{2}{*}{ Patient No. } & \multirow{2}{*}{ Sex/Age (yr) } & \multirow{2}{*}{ Location } & \multirow{2}{*}{ Size } & \multirow{2}{*}{ Mitosis } & \multirow{2}{*}{ Ki-67 LI } & \multirow{2}{*}{$\mathrm{PHH} 3 \mathrm{Ml}$} & \multicolumn{2}{|c|}{ Risk of tumor progression (\%) } \\
\hline & & & & & & & AFIP criteria & PHH3 Ml applied \\
\hline 1 & $M / 51$ & Duodenum & 4.5 & 2 & 10 & 14 & Low (8.3) & High (50) \\
\hline 2 & $\mathrm{M} / 48$ & Jejunum & 3.0 & 2 & 4 & 6 & Low (4.3) & High (73) \\
\hline 3 & $\mathrm{M} / 47$ & lleum & 3.9 & 2 & 8 & 10 & Low (4.3) & High (73) \\
\hline 4 & $M / 56$ & Rectum & 2.1 & 4 & 3 & 7 & Low (8.5) & High (52) \\
\hline 5 & $\mathrm{M} / 67$ & Stomach & 3.0 & 1 & 4 & 8 & Very low (1.9) & Moderate (16) \\
\hline 6 & $M / 57$ & Stomach & 2.5 & 2 & 3 & 7 & Very low (1.9) & Moderate (16) \\
\hline 7 & $\mathrm{M} / 57$ & Stomach & 4.5 & 3 & 6 & 6 & Very low (1.9) & Moderate (16) \\
\hline 8 & $F / 67$ & Stomach & 2.9 & 4 & 5 & 10 & Very low (1.9) & Moderate (16) \\
\hline 9 & $\mathrm{M} / 64$ & Stomach & 2.7 & 5 & 5 & 14 & Very low (1.9) & Moderate (16) \\
\hline 10 & $\mathrm{M} / 64$ & Stomach & 2.2 & 5 & 3 & 6 & Very low (1.9) & Moderate (16) \\
\hline
\end{tabular}

PHH3 MI, phosphohistone H3 mitotic index; AFIP, Armed Forces Institute of Pathology; Ki-67 LI, Ki-67 labeling index.

PHH3 MI ( $\mathrm{r}=0.810, \mathrm{p}<.001)$. Mitotic counts correlated with both the Ki-67 LI and PHH3 MI, but the correlation with PHH3 MI was slightly higher. The PHH3 MI also correlated with Ki$67 \mathrm{LI}(\mathrm{r}=0.703, \mathrm{p}<.001)$. The inter-observer agreement among the three participating pathologists was high for both the mitotic count (interclass correlation coefficient, $0.974 ; 95 \%$ confidence interval [CI], 0.962 to 0.983 ) and the PHH3 MI (interclass correlation coefficient, $0.940 ; 95 \% \mathrm{CI}, 0.912$ to 0.961 ). When the PHH3 MI replaced mitotic counts in the AFIP risk restratification analysis, the $\mathrm{PHH} 3 \mathrm{MI}$ imposed a higher risk among the 10 cases compared to using mitotic counts in the risk stratification (Table 3); 4 of these 10 cases were from extragastric sites (duodenum, jejunum, ileum, and rectum). This change in risk tended to be greater in extragastric GISTs when compared to gastric GISTs.

In all 10 discrepant cases, the $\mathrm{H} \& \mathrm{E}-$ and $\mathrm{PHH} 3$-immunostained slides were thoroughly reviewed. In case No. 1, the highest proliferative area on the $\mathrm{H} \& \mathrm{E}$ - and the $\mathrm{PHH} 3$-immunostained slides were not well matched. In cases Nos. 3, 5, 6, and 8 , many inflammatory cells were admixed with tumor cells which may have artifactually inflated the PHH3 MI. In cases Nos. 7 , 9, and 10, diffuse degeneration of tumor cells was observed on the H\&E-stained slides rendering mitoses difficult to identify. In contrast, on the $\mathrm{PHH} 3$-immunostained slides, the darkly stained PHH3-positive cells were easy to identify even at low magnification (Fig. 1). Degenerated mitotic tumor cells, which are difficult to identify on H\&E-immunostained slides, were also highlighted as dark brown figures on the $\mathrm{PHH} 3$-immunostained slides (Fig. 2). The last case (case No. 2) was stratified in the low risk of aggressive behavior group by both the $\mathrm{NIH}$ and the AFIP criteria when diagnosed in 2012. In 2014, the patient was found to have a metastasis in the liver. The primary tumor was located in the jejunum with a size of $3 \mathrm{~cm}$ and a mi- totic count of 2 per $50 \mathrm{HPFs}$. The PHH3 MI for this case was 6 per 50 HPFs and the mean PHH3 MI among the three observers was 7 per 50 HPFs (Fig. 3) placing this tumor into the high risk group in the restratification of the AFIP criteria using the PHH3 MI. This correlates with an approximately $73 \%$ chance of malignant behavior.

\section{DISCUSSION}

To explore the diagnostic utility of the $\mathrm{PHH} 3$ immunohistochemical stain as an ancillary mitotic marker in GIST, a comparison was made between mitotic counts, Ki-67 LI and PHH3 MI in 77 primary GISTs. The PHH3 MI correlated very well with mitotic counts and the inter-observer correlation coefficient among the 3 pathologists was also high. However, in cases with poor fixation, abundant lymphocytes, or degeneration of tumor cells, a discrepancy between risk categorization based on mitotic counts and the PHH3 MI was observed. This study supports the use of $\mathrm{PHH} 3$ as a helpful adjunct strategy for the efficient analysis of mitotic figures in GISTs.

$\mathrm{Ki}-67$ has been widely used as a proliferative marker in many different tumors, including GISTs. ${ }^{19-21}$ However, Ki-67 is expressed throughout the cell cycle except in the G0 phase. Therefore, there is no assurance that Ki-67-positive cells are actually undergoing mitosis. ${ }^{22}$ In contrast, $\mathrm{PHH} 3$, a recently introduced mitosis-specific marker, only highlights cells in the late G2 and M phases. ${ }^{23,24}$ Discrepancies have been found in the correlation between $\mathrm{PHH} 3$ and $\mathrm{Ki}-67$ expression in various tumors for the above reason. ${ }^{16,17}$ The robust positive correlation between $\mathrm{PHH} 3$ and mitotic counts has been well established in many different tumors and benign tissues. ${ }^{9-11,14,15,18,25}$

In the present study, the PHH3 MI showed a higher correlation with mitotic counts than Ki-67 LI. The PHH3 MI also 

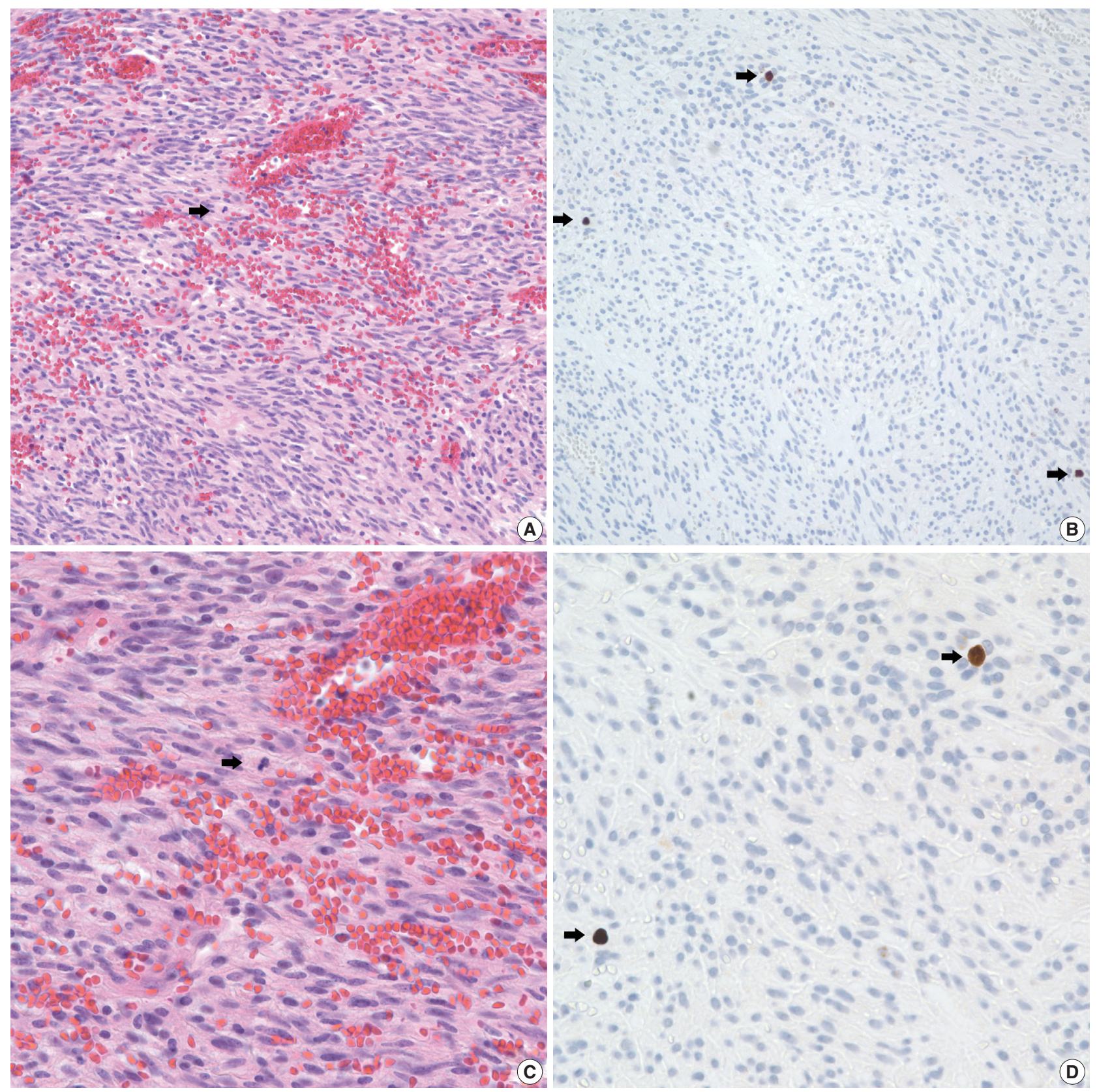

Fig. 1. A comparison of hematoxylin and eosin $(A, C)$ and phophohistone $H 3(P H H 3)$ immunohistochemistry $(B, D)$ in mitotic detection (arrows). Mitotic figures (arrows) are easily and quickly recognized with $\mathrm{PHH} 3$ immunohistochemistry.

correlated with Ki-67 LI in the current study. Interclass correlation coefficients among 3 observers for counting mitoses with either staining method were high. The interclass correlation coefficient was slightly higher for counting mitoses than for $\mathrm{PHH} 3$ MI in this study. In a series of 92 thin melanoma cases, interobserver agreement was higher using the $\mathrm{PHH} 3 \mathrm{MI}$ than with mitotic counts. ${ }^{26}$ In the present study, in some cases with poor fixation the immunoreactivity of the $\mathrm{PHH} 3$ antibody had vari- able staining intensity making accurate quantification of $\mathrm{PHH} 3$ MI difficult. Higher inter-observer agreement for $\mathrm{PHH} 3 \mathrm{MI}$ may be achieved with a standardized high quality immunostaining method and by consensus.

To assess the substitutability of $\mathrm{PHH} 3$ analysis for mitotic counts, the cases were restratified according to the PHH3 MI in accordance with the established AFIP criteria. Considering recent data suggesting tumor location can affect the malignant 

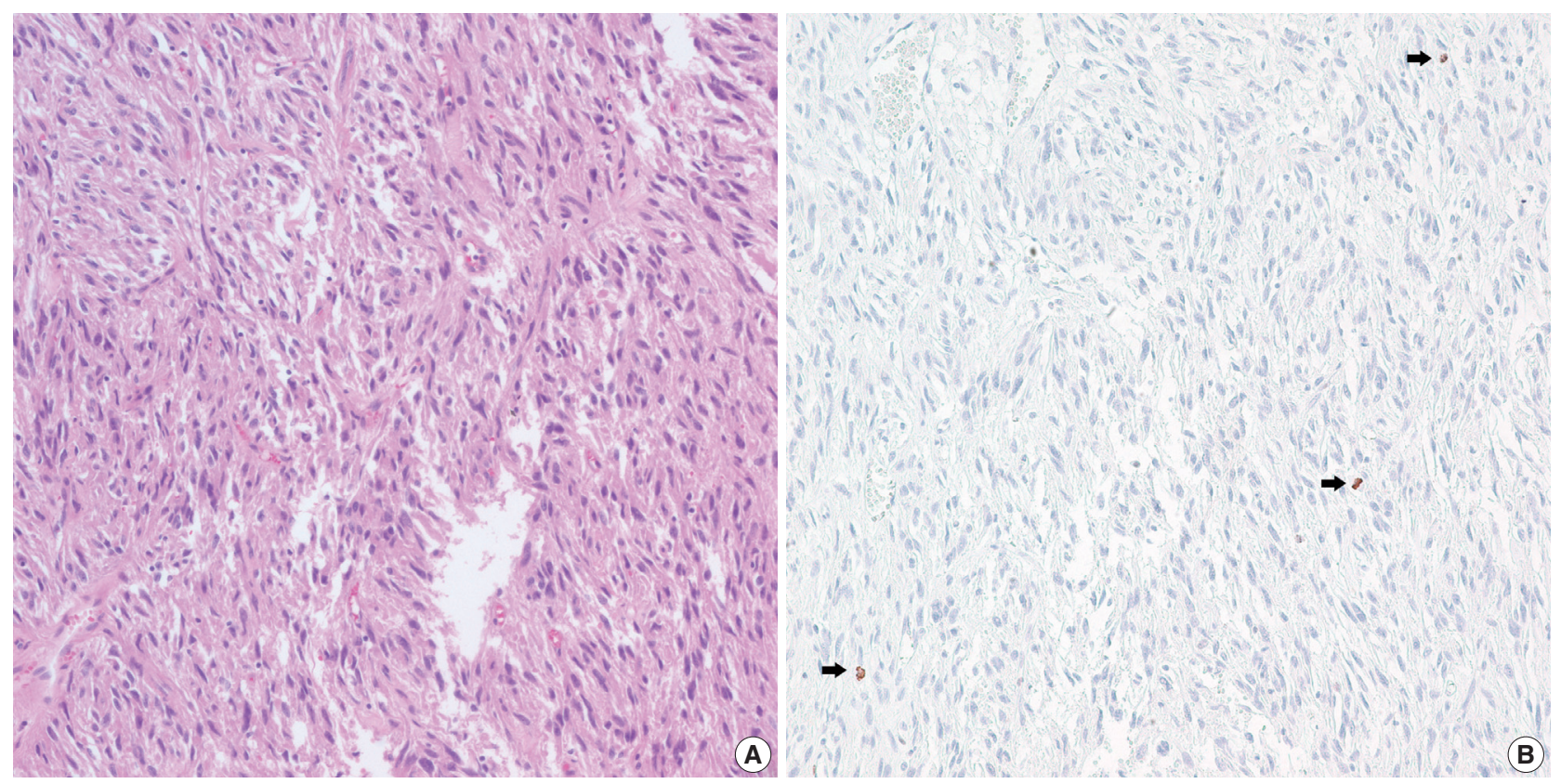

Fig. 2. Hematoxylin and eosin $(H \& E)(A)$ and phophohistone $H(P H H 3)$ immunohistochemistry $(B)$ in a case with delayed fixation. H\&E shows no mitotic figures whereas $\mathrm{PHH} 3$ shows three mitotic figures in the same field (arrows).

potential of GISTs, the AFIP criteria were used to analyze all cases. The 10 cases showing a change in risk stratification after applying the PHH3 MI were reviewed. The use of the $\mathrm{PHH} 3$ MI led to a higher risk categorization when compared to the use of mitotic counts in all 10 cases. These cases showed poor specimen fixation, abundant lymphocytes, or degeneration of tumor cells. Poor fixation of tumor cells causes difficulty in counting mitotic figures precisely. Abundant lymphocytes around tumor cells may also confuse pathologists. $\mathrm{PHH} 3$ is positive in mitotic tumor cells and in interphase nuclei and mitotic lymphocytes. Therefore, careful counting is needed and pathologists may overestimate the $\mathrm{PHH} 3 \mathrm{MI}$ in cases with abundant lymphocytic infiltration. In fact, several published reports indicate the $\mathrm{PHH} 3 \mathrm{MI}$ is higher than mitotic counts in various tumor types likely due to this reason. $7,9,11,14,15,26$ The $\mathrm{PHH} 3$ immunostain highlights mitotic cells as distinct dark brown figures rendering their identification much simpler and easier than searching for mitotic figures and allows rapid detection of the most proliferative area of a lesion.

In the current study, a direct relationship between the $\mathrm{PHH} 3$ $\mathrm{MI}$ and disease-free survival was not proven. Kim et al. ${ }^{19} \mathrm{dem}$ onstrated a significant correlation between recurrence-free survival and PHH3 MI, but failed to prove the independent prognostic significance of the $\mathrm{PHH} 3 \mathrm{MI}$ in GISTs. Interestingly, we found a case of a jejunum GIST stratified as low risk of malignant potential according to both the NIH and AFIP criteria.
However, this lesion metastasized to the liver 2 years after the initial diagnosis. The mitotic count of the primary GIST was 2 per 50 HPFs and the PHH3 MI and mean PHH3 MI among the 3 observers was 6 per $50 \mathrm{HPFs}$ and 7 per $50 \mathrm{HPFs}$, respectively. When the PHH3 MI was applied to risk stratification in place of mitotic counts, the case was reclassified into the high risk group ( $73 \%$ chance of malignant behavior). Therefore, more attention should be given to cases with a higher PHH3 MI. These cases may warrant risk recategorization. To obtain reliable results regarding the $\mathrm{PHH} 3 \mathrm{MI}$ as an independent prognostic factor in GISTs, a large-scale study is needed with a prolonged follow-up period.

In summary, the PHH3 MI correlated satisfactorily with mitotic counts even when compared to the Ki-67 LI in GISTs. Overall, inter-observer agreement for mitotic counts and the PHH3 MI was both high. The PHH3 immunostain enables pathologists to identify mitotic figures easily and quickly. Furthermore, in cases with inadequate fixation, quantification of the $\mathrm{PHH} 3 \mathrm{MI}$ was superior to counting mitotic figures. We believe the $\mathrm{PHH} 3 \mathrm{MI}$ is a viable adjunct strategy to use when assessing the mitotic rates in GISTs.

\section{Conflicts of Interest}

No potential conflict of interest relevant to this article was reported. 

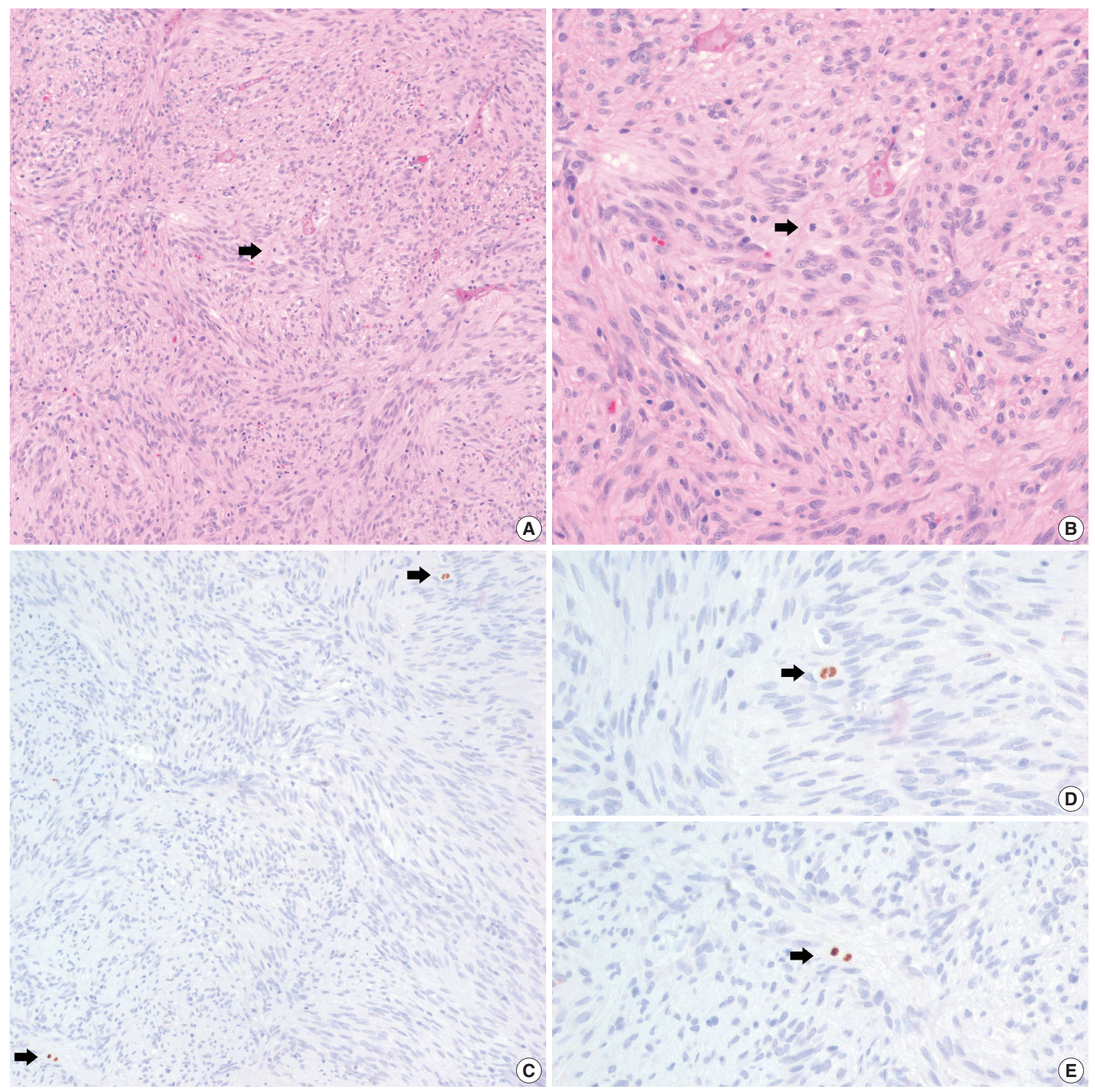

Fig. 3. Mitosis and PHH3 positive cells in a patient with distant metastasis. Hematoxylin and eosin (H\&E) staining shows a single mitotic figure. (B) H\&E at higher magnification. (C) Phophohistone H3 (PHH3) immunohistochemistry highlights two mitotic figures in the same field (arrows). (D, E) PHH3 at higher magnification.

\section{REFERENCES}

1. Miettinen M, Lasota J. Gastrointestinal stromal tumors: review on morphology, molecular pathology, prognosis, and differential diagnosis. Arch Pathol Lab Med 2006; 130: 1466-78.

2. Corless CL, Fletcher JA, Heinrich MC. Biology of gastrointestinal stromal tumors. J Clin Oncol 2004; 22: 3813-25.

3. Franquemont DW. Differentiation and risk assessment of gastroin- testinal stromal tumors. Am J Clin Pathol 1995; 103: 41-7.

4. Fletcher CD, Berman JJ, Corless C, et al. Diagnosis of gastrointestinal stromal tumors: a consensus approach. Hum Pathol 2002; 33: $459-65$.

5. Miettinen M, Sobin LH, Lasota J. Gastrointestinal stromal tumors of the stomach: a clinicopathologic, immunohistochemical, and molecular genetic study of 1765 cases with long-term follow-up. Am J Surg Pathol 2005; 29: 52-68. 
6. Miettinen M, Makhlouf H, Sobin LH, Lasota J. Gastrointestinal stromal tumors of the jejunum and ileum: a clinicopathologic, immunohistochemical, and molecular genetic study of 906 cases before imatinib with long-term follow-up. Am J Surg Pathol 2006; 30: 47789.

7. Tapia C, Kutzner H, Mentzel T, Savic S, Baumhoer D, Glatz K. Two mitosis-specific antibodies, MPM-2 and phospho-histone H3 (Ser28), allow rapid and precise determination of mitotic activity. Am J Surg Pathol 2006; 30: 83-9.

8. Agaimy A. Gastrointestinal stromal tumors (GIST) from risk stratification systems to the new TNM proposal: more questions than answers? A review emphasizing the need for a standardized GIST reporting. Int J Clin Exp Pathol 2010; 3: 461-71.

9. Hendzel MJ, Wei Y, Mancini MA, et al. Mitosis-specific phosphorylation of histone $\mathrm{H} 3$ initiates primarily within pericentromeric heterochromatin during G2 and spreads in an ordered fashion coincident with mitotic chromosome condensation. Chromosoma 1997; 106: 348-60.

10. Ribalta T, McCutcheon IE, Aldape KD, Bruner JM, Fuller GN. The mitosis-specific antibody anti-phosphohistone- $\mathrm{H} 3$ ( $\mathrm{PHH} 3$ ) facilitates rapid reliable grading of meningiomas according to WHO 2000 criteria. Am J Surg Pathol 2004; 28: 1532-6.

11. Kim YJ, Ketter R, Steudel WI, Feiden W. Prognostic significance of the mitotic index using the mitosis marker anti-phosphohistone $\mathrm{H} 3$ in meningiomas. Am J Clin Pathol 2007; 128: 118-25.

12. Fukushima S, Terasaki M, Sakata K, et al. Sensitivity and usefulness of anti-phosphohistone-H3 antibody immunostaining for counting mitotic figures in meningioma cases. Brain Tumor Pathol 2009; 26: 51-7.

13. Bossard C, Jarry A, Colombeix C, et al. Phosphohistone H3 labelling for histoprognostic grading of breast adenocarcinomas and computer-assisted determination of mitotic index. J Clin Pathol 2006; 59: 706-10.

14. Nasr MR, El-Zammar O. Comparison of pHH3, Ki-67, and survivin immunoreactivity in benign and malignant melanocytic lesions. Am J Dermatopathol 2008; 30: 117-22.

15. Veras E, Malpica A, Deavers MT, Silva EG. Mitosis-specific marker phospho-histone $\mathrm{H} 3$ in the assessment of mitotic index in uterine smooth muscle tumors: a pilot study. Int J Gynecol Pathol 2009; 28:
316-21.

16. Tsuta K, Liu DC, Kalhor N, Wistuba II, Moran CA. Using the mitosis-specific marker anti-phosphohistone $\mathrm{H} 3$ to assess mitosis in pulmonary neuroendocrine carcinomas. Am J Clin Pathol 2011; 136: 252-9.

17. Takahashi H, Murai Y, Tsuneyama K, et al. Overexpression of phosphorylated histone $\mathrm{H} 3$ is an indicator of poor prognosis in gastric adenocarcinoma patients. Appl Immunohistochem Mol Morphol 2006; 14: 296-302.

18. Nakashima S, Shiozaki A, Ichikawa D, et al. Anti-phosphohistone $\mathrm{H} 3$ as an independent prognostic factor in human esophageal squamous cell carcinoma. Anticancer Res 2013; 33: 461-7.

19. Kim A, Im DH, Kim K, et al. Usefulness of anti-phosphohistone H3 immunoreactivity to determine mitotic rate in gastrointestinal stromal tumors. Basic Appl Pathol 2012; 5: 91-7.

20. Carrillo R, Candia A, Rodriguez-Peralto JL, Caz V. Prognostic significance of DNA ploidy and proliferative index (MIB-1 index) in gastrointestinal stromal tumors. Hum Pathol 1997; 28: 160-5.

21. Toquet C, Le Neel JC, Guillou L, et al. Elevated (> or $=10 \%$ ) MIB-1 proliferative index correlates with poor outcome in gastric stromal tumor patients: a study of 35 cases. Dig Dis Sci 2002; 47: 2247-53.

22. Filiz G, Yalçinkaya O, Gürel S, Yerci O, Memik F. The relationship between MIB-1 proliferative activity and mitotic index in gastrointestinal stromal tumors. Hepatogastroenterology 2007; 54: 438-41.

23. Gerdes J, Lemke H, Baisch H, Wacker HH, Schwab U, Stein H. Cell cycle analysis of a cell proliferation-associated human nuclear antigen defined by the monoclonal antibody Ki-67. J Immunol 1984; 133: 1710-5.

24. Juan G, Traganos F, James WM, et al. Histone H3 phosphorylation and expression of cyclins A and B1 measured in individual cells during their progression through G2 and mitosis. Cytometry 1998; 32: 71-7.

25. Brenner RM, Slayden OD, Rodgers WH, et al. Immunocytochemical assessment of mitotic activity with an antibody to phosphorylated histone $\mathrm{H} 3$ in the macaque and human endometrium. Hum Reprod 2003; 18: 1185-93.

26. Schimming TT, Grabellus F, Roner M, et al. $\mathrm{pHH} 3$ immunostaining improves interobserver agreement of mitotic index in thin melanomas. Am J Dermatopathol 2012; 34: 266-9. 